\title{
HARDY SPACES OF CLOSE-TO-CONVEX FUNCTIONS AND THEIR DERIVATIVES \\ BY
}

FINBARR HOLLAND AND JOHN B. TWOMEY

ABSTRACT. Let $f(z)=\Sigma_{1}^{\infty} a_{n} z^{n}$ be close-to-convex on the unit disc. It is shown that (a) if $\lambda>0$, then $f$ belongs to the Hardy space $H^{\lambda}$ if and only if $\sum n^{\lambda-2}\left|a_{n}\right|^{\lambda}$ is finite and that (b) if $0<\lambda<1$, then $f^{\prime} \in H^{\lambda}$ if and only if either $\Sigma n^{2 \lambda-2}\left|a_{n}\right|^{\lambda}$ or, equivalently, $\int_{0}^{1} M^{\lambda}\left(r, f^{\prime}\right) d r$ is convergent. It is noted that the first of these results does not extend to the full class of univalent functions and that the second is best possible in a number of different senses.

\section{Introduction and summary of results. Let}

$$
f(z)=\sum a_{n} z^{n}=\sum_{1}^{\infty} a_{n} z^{n}
$$

be regular on the unit disc $\Delta=\{z:|z|<1\}$ and, for $0 \leqslant r<1$, define the family of means

$$
\left\{M_{\lambda}(r): 0<\lambda \leqslant \infty\right\}
$$

by

$$
M_{\lambda}(r)=M_{\lambda}(f)=M_{\lambda}(r, f)=\left(\frac{1}{2 \pi} \int\left|f\left(r e^{i \theta}\right)\right|^{\lambda} d \theta\right)^{1 / \lambda},
$$

with the usual convention applying here and below when $\lambda=\infty$. Thus, for instance,

$$
M_{\infty}(r, f)=\max \{|f(z)|:|z|=r\},
$$

which for notational convenience we write variously as $M(r, f), M(r)$ or $M(f)$. (Here too and throughout the paper we adopt the practice of not displaying limits on (i) sums that are over the set of positive integers; (ii) integrals with respect to $d \theta$ that are over $(0,2 \pi)$; and (iii) integrals with respect to $d t$ that are over $(0,1)$.)

Received by the editors March 9, 1978.

AMS (MOS) subject classifications (1970). Primary 30A32, 30A78; Secondary 30A76.

Key words and phrases. Membership of the Hardy space $H^{\lambda}$, close-to-convex functions, functions with positive real part, Riesz products, Taylor coefficients. 
Fix $\lambda>0$ and consider the following propositions:

$$
\sup \left\{M_{\lambda}(r, f): 0<r<1\right\}<\infty,
$$

i.e., $f$ belongs to the Hardy space $H^{\lambda}$;

$$
\left(\int M^{\lambda}(t, f) d t\right)^{1 / \lambda}<\infty
$$

i.e., the maximum modulus function $M(f)$ is an element of the Lebesgue space $L^{\lambda}(0,1)$;

$$
\left(\sum n^{\lambda-2}\left|a_{n}\right|^{\lambda}\right)^{1 / \lambda}<\infty
$$

i.e., the series $\sum n^{\lambda-2}\left|a_{n}\right|^{\lambda}$ is convergent if $0<\lambda<\infty$, and the sequence ( $n a_{n}$ ) is bounded if $\lambda=\infty$;

the derivative $f^{\prime}$ belongs to $H^{\mu}$, where $\mu=\lambda /(1+\lambda)$.

Of the very many significant results established by Hardy and Littlewood involving these statements, we recall here only the following:

TheOREM A. If $0<\lambda \leqslant \infty$, then (1.1) $\Rightarrow(1.2)$.

TheOREM B. If $0<\lambda \leqslant 2$, then $(1.1) \Rightarrow(1.3)$.

TheOrem C. If $2 \leqslant \lambda<\infty$, then (1.3) $\Rightarrow$ (1.1).

TheOREM D. If $0<\lambda \leqslant \infty$, then (1.4) $\Rightarrow(1.1)$.

Aside from the trivial fact that (1.1) and (1.2) say the same thing if $\lambda=\infty$, the converse of each of these theorems is false in general. (A proof of this fact, together with a good discussion of these theorems can be found in [4].) However, restricted converses of Theorems A, B, and C are known. Thus, for instance, if $f$ is univalent on $\Delta$, then (1.1) and (1.2) are equivalent for all $\lambda>0$, and (1.1) and (1.3) for $\lambda \dot{\epsilon}[1,2]$. Again if $f \in \mathscr{K}$, the class of close-to-convex functions defined below, then (1.1) $\Leftrightarrow(1.3)$ if $1<\lambda<\infty$. (These and related observations are noted in our paper [8].) Whereas, as Clunie and Pommerenke have shown in [3], the implication "(1.1) $\Rightarrow(1.3)$ " also holds when $f \in \mathscr{K}$ and $\lambda=\infty$, the opposite conclusion is false, as the example

$$
f(z)=\sum n^{-1} z^{n}, \quad z \in \Delta,
$$

shows: as in the general case, the most we can conclude is that

$$
f \in \bigcap\left\{H^{\lambda}: 0<\lambda<\infty\right\} .
$$

One purpose of the present paper is to complete a line of study initiated in [8] about the class $\mathscr{K}$; another is to obtain converses of Theorems A and B for the class $\mathcal{K}^{\prime}=\left\{g^{\prime} ; g \in \mathscr{K}\right\}$ when $0<\lambda<1$, and yet another is to 
establish that the converse of Theorem D holds if $f$ is starlike, but not if $f \in \mathcal{K}$. We proceed to formulate our principal results.

THEOREM 1. Let $f(z)=\Sigma a_{n} z^{n} \in \mathscr{K}$ and $0<\lambda<\infty$. Then

$$
f \in H^{\lambda} \Leftrightarrow \sum n^{\lambda-2}\left|a_{n}\right|^{\lambda}<\infty .
$$

In view of the known results mentioned above, Theorem 1 follows once we show that (1.3) forces (1.1) when $0<\lambda<1$; and this we do in $\$ 4$.

TheOREM 2. Let $f(z)=\sum a_{n} z^{n} \in \mathscr{K}$ and $0<\lambda<1$. Then the following statements are equivalent.

(a) $f^{\prime} \in H^{\lambda}$.

(b) $M\left(f^{\prime}\right) \in L^{\lambda}(0,1)$.

(c) $\sum n^{2 \lambda-2}\left|a_{n}\right|^{\lambda}<\infty$.

This theorem is best possible in a number of senses. First of all, we observe that (b) $\Rightarrow$ (a) if $\lambda=1$. In fact, a good deal more can be said, namely

THeOREM 3. Let $f$ be starlike. Then no matter how slowly $M\left(r, f^{\prime}\right)$ tends to infinity as $r \rightarrow 1^{-}$we cannot, in general, infer that $f^{\prime} \in H^{1}$.

Since it is true, generally, that (c) $\Rightarrow$ (a) if $\lambda \geqslant 2$, it is tempting to speculate that this also occurs if $1 \leqslant \lambda<2$ and $f \in \mathscr{K}$. But the next result shows that this is not the case.

TheOREM 4. Suppose $1 \leqslant \lambda<2$. Then there is a starlike function $f$, whose Taylor coefficients $a_{n}$ are nonnegative, such that (c) obtains and yet $f^{\prime} \notin H^{\lambda}$.

There remains the possibility that (a) $\Rightarrow$ (c) if $\lambda \geqslant 1$ and $f \in \mathscr{K}$. This is certainly true if $1 \leqslant \lambda \leqslant 2$, by Theorem $B$. However, it is false if $2<\lambda<\infty$. To see this, let $2<\lambda<\infty, 0<\alpha=1-2 / \lambda$ and consider the function

$$
f(z)=z+\left(2^{\alpha}-1\right) \sum 2^{-(1+\alpha) m} z^{2^{m}} \equiv \sum a_{n} z^{n} .
$$

Then

$$
\left|f^{\prime}(z)-1\right| \leqslant \sum_{2}^{\infty} n a_{n}=1 \quad \text { for all } z \in \Delta .
$$

Hence (a) holds and $f$ is starlike [2], but (c) is false.

Finally, we remark that Theorem 2 does not extend to the full class of univalent functions. Indeed, whereas for such functions statements (b) and (c) hold if $0<\lambda<1 / 3$, Lohwater, Piranian and Rudin have shown in [11] (cf. also [1]) that a sequence $(n(p))$ of positive integers can be chosen so that the function

$$
f(z)=\int_{0}^{z} \exp \left\{\frac{1}{2} \sum w^{n(p)}\right\} d w, \quad z \in \Delta,
$$


is univalent on $\Delta$, belongs to $H^{\infty}$ and yet its derivative has unbounded characteristic. Since $(n(p))$ can also be selected (cf. $[4$, p. 87]) to meet the requirement

$$
\sum r^{n(p)}=O(\varphi(r)) \quad(r \rightarrow 1)
$$

for a given $\varphi(r)$ increasing to infinity as $r \rightarrow 1$, it follows that we have a univalent function $f$ such that $M\left(r, f^{\prime}\right)$ increases arbitrarily slowly to infinity and

$$
\sup \left\{\int \log ^{+}\left|f^{\prime}\left(r e^{i \theta}\right)\right| d \theta: 0 \leqslant r<1\right\}=+\infty .
$$

Thus, for no $\lambda>0$ is it true that (a) follows from (b) if $f$ is assumed to be merely univalent.

Theorem 4 shows that the implication "(c) $\Rightarrow$ (a)" fails if $f$ is univalent and $1 \leqslant \lambda<2$. The examples just discussed have absolutely convergent power series on $|z|=1$-since they are bounded and have nonnegative Taylor coefficients-and hence satisfy (c), by Hölder's inequality, if $0<\lambda<1$. Thus the implication "(c) $\Rightarrow$ (a)" fails also if $0<\lambda<1$.

Theorems 2, 3 and 4 are proved in $\$ 5$ and in the last section we prove the following results.

Theorem 5. Suppose $f \in \mathcal{K} \cap H^{\lambda}$, where $0<\lambda<\infty$. Then $f^{\prime} \in H^{\nu}$, where $0<\nu<\mu=\lambda /(1+\lambda)$; but $f^{\prime} \notin H^{\mu}$ in general.

THEOREM 6. If $f$ is starlike and $0<\lambda<\infty$, then $f \in H^{\lambda}$ if and only if $f^{\prime} \in H^{\mu}$, where $\mu=\lambda /(1+\lambda)$.

2. Definitions and notations. For a given regular function $f(z)=\sum a_{n} z^{n}$ we set

$$
P(r)=P(r, f)=\sum\left|a_{n}\right| r^{n}, \quad 0<r<1 .
$$

We write $\mathcal{P}$ for the class of regular functions of the form

$$
h(z)=1+\sum c_{n} z^{n}, \quad z \in \Delta,
$$

which have positive real part on $\Delta$.

A regular function

$$
f(z)=\sum a_{n} z^{n}
$$

is said to belong to the class $\mathcal{S}^{*}$ of starlike functions if and only if $f$ is univalent on $\Delta$ and the image $f(\Delta)$ contains the line segment $[0, W]$ whenever it contains $W$. It can be shown that

$$
f \in \mathcal{S}^{*} \Leftrightarrow F(z)=z f^{\prime}(z) / f(z) \in \mathcal{P} .
$$


For each $f \in \delta^{*}$ the limit

$$
\lim _{r \rightarrow 1} \frac{\log M(r, f)}{-\log (1-r)}=\alpha(f)
$$

exists and $0<\alpha(f)<2[12] ; \alpha(f)$ is called the order of $f$.

A function $f$ of the form (2.1) is called close-to-convex if there is a starlike function $g$ and a function $h \in \mathscr{P}$ such that

$$
z f^{\prime}(z)=g(z) h(z), \quad z \in \Delta .
$$

The class $\mathscr{K}$ of such functions was introduced by Kaplan [10] and shown by him to consist of univalent functions. It is clear that $\mathcal{S}^{*} \subset \mathcal{K}$.

3. Some preliminary lemmas.

We begin by establishing a result about the class of univalent functions.

LEMMA 1. If $f(z)=\sum a_{n} z^{n}$ is univalent on $\Delta$, then

$$
r P(r, f)<16 P\left(r^{2}, f\right), \quad 0<r<1 .
$$

Proof. Let $0 \leqslant r<1$. Then, by Schwarz's inequality,

$$
\begin{aligned}
r P^{\prime}(r) & =\sum n\left|a_{n}\right| r^{n} \\
& <\left(\sum n\left|a_{n}\right|^{2} r^{n+1}\right)^{1 / 2}(1-r)^{-1} \\
& =\left(r A\left(r^{1 / 2}\right)\right)^{1 / 2}(1-r)^{-1},
\end{aligned}
$$

where $\pi A(r)$ is the area of $f(\{z:|z|<r\})$. Since

$$
A(r)<M^{2}(r)<P^{2}(r)
$$

and the function $t \rightarrow(1-t)^{2} t^{-1} M(t)$ decreases on $(0,1)[7]$, it follows that

$$
\begin{aligned}
r P^{\prime}(r) & <M\left(r^{1 / 2}\right) r^{1 / 2}(1-r)^{-1} \\
& <M(r) \frac{1+r^{1 / 2}}{1-r^{1 / 2}}<P(r) \frac{1+r^{1 / 2}}{1-r^{1 / 2}} .
\end{aligned}
$$

Hence the function

$$
t \rightarrow\left(1-t^{1 / 2}\right)^{4} t^{-1} P(t)
$$

is also decreasing on $(0,1)$. The stated result is now an easy consequence of this.

We refer to [3] for a proof of the next result.

Lemma 2. Let $f \in \mathscr{K}$. Then, for every pair of functions $g \in \mathcal{S}^{*}, h \in \mathcal{P}$ involved in the factorization (2.3), we have

$$
\int\left|g\left(r e^{i \theta}\right)\right| \operatorname{Re} h\left(r e^{i \theta}\right) d \theta<2 \pi M(r, f)
$$

if $0<r<1$. 
The same source also contains all the ingredients for a proof of Lemma 3, but, for convenience, we give some of the details.

Lemma 3. Let $f(z)=\sum a_{n} z^{n} \in \mathscr{K}$. Then, if $0<r<1$,

$$
n\left|a_{n}\right| r^{n} \leqslant 4 P(r, f), \quad n=1,2 \ldots
$$

Proof. Our starting point is the inequality

$$
\begin{aligned}
n\left|a_{n}\right| \leqslant & \frac{1}{\pi r^{n}} \int\left|g\left(r e^{i \theta}\right)\right| \operatorname{Re} h\left(r e^{i \theta}\right) d \theta \\
& +\frac{1}{2 \pi r^{n}}\left|\int \overline{g\left(r e^{i \theta}\right)} h\left(r e^{i \theta}\right) e^{i n \theta} d \theta\right|
\end{aligned}
$$

taken from [3], where $g \in \mathcal{S}^{*}, h \in \mathcal{P}$ and (2.3) holds.

By Lemma 2, the first term on the right of (3.1) is dominated by $2 r^{-n} M(r)$. The second term can be expressed as $r^{-2 n}\left|J_{n}(2, r)\right|$, where

$$
J_{n}(2, r)=\frac{1}{2 \pi} \int_{|z|=r} z^{n+1} f^{\prime}(z) e^{-2 i \arg g(z)} \frac{d z}{i z} .
$$

Now

$$
J_{n}(2, r)=\frac{1}{\pi} \int_{|z|=r} f_{n}(z) e^{-2 i \arg g(z)} \operatorname{Re} G(z) \frac{d z}{i z}
$$

after an integration by parts, where

$$
G(z)=z g^{\prime}(z) / g(z) \text { and } f_{n}(z)=\int_{0}^{z} w^{n} f^{\prime}(w) d w, \quad z \in \Delta .
$$

Clearly

$$
\left|J_{n}(2, r)\right|<2 M\left(r, f_{n}\right)
$$

since $G \in \mathscr{P}$. Putting these facts together, we see that

$$
n\left|a_{n}\right| r^{n}<2 M(r)+2 r^{-n} M\left(r, f_{n}\right)<4 P(r),
$$

as asserted.

4. Proof of Theorem 1. The case $\lambda \geqslant 1$ was completely disposed of in [8]. As far as the remaining case, $0<\lambda<1$, is concerned, it is enough, in view of Theorem B, to establish that the finiteness of the series $\sum n^{\lambda-2}\left|a_{n}\right|^{\lambda}$ qualifies $f$ for membership of $H^{\lambda}$; and this is an easy consequence of the following more general theorem.

THEOREM 7. Let $f(z)=\sum a_{n} z^{n} \in \mathscr{K}, 0<x<1$ and $0<y<1$. Then there is a positive constant $C$, depending only on $x$ and $y$, such that

$$
\int(P(t, f))^{x}(1-t)^{-y} d t<C \sum n^{x+y-2}\left|a_{n}\right|^{x} .
$$


Proof. For $s>0$ and $0<r<1$, we write

$$
P_{s}(r)=\sum n^{s-1}\left|a_{n}\right|^{s} r^{n},
$$

and, using Hölder's inequality in a straightforward manner, we find that

$$
\begin{aligned}
P(r) & =\sum\left|a_{n}\right| r^{n}=\sum\left(n^{x-1}\left|a_{n}\right|^{x} r^{n}\right)^{x}\left(n^{x}\left|a_{n}\right|^{1+x} r^{n}\right)^{1-x} \\
& <\left(\sum n^{x-1}\left|a_{n}\right|^{x} r^{n}\right)^{x}\left(\sum n^{x}\left|a_{n}\right|^{1+x} r^{n}\right)^{1-x} \\
& =\left(P_{x}(r)\right)^{x}\left(P_{1+x}(r)\right)^{1-x} .
\end{aligned}
$$

Now

$$
\begin{aligned}
P_{1+x}\left(r^{2}\right) & <P_{1+x}\left(r^{1+x}\right)=\sum\left(n\left|a_{n}\right| r^{n}\right)^{x}\left|a_{n}\right| r^{n} \\
& <4^{x}(P(r))^{1+x}
\end{aligned}
$$

by Lemma 3 . Hence

$$
P\left(r^{2}\right)<\left(P_{x}\left(r^{2}\right)\right)^{x}\left(P_{1+x}\left(r^{2}\right)\right)^{1-x}<C(x)\left(P_{x}\left(r^{2}\right)\right)^{x}(P(r))^{1-x^{2}},
$$

where, here and immediately following, $C(x)$ stands for a positive constant, depending only on $x$, and not necessarily the same at each occurrence.

Appealing now to Lemma 1, we infer that, if $r>\frac{1}{2}$, say, then

$$
P\left(r^{2}\right)<C(x)\left(P_{x}\left(r^{2}\right)\right)^{x}\left(P\left(r^{2}\right)\right)^{1-x^{2}},
$$

and so

$$
(P(t))^{x}<C(x) P_{x}(t)
$$

if $t \in\left[\frac{1}{4}, 1\right]$. It now follows that

$$
\begin{aligned}
\int(P(t))^{x}(1-t)^{-y} d t & <C(x) \int P_{x}(t)(1-t)^{-y} d t \\
& =C(x) \sum n^{x-1}\left|a_{n}\right|^{x} \int t^{n}(1-t)^{-y} d t \\
& =C(x) \sum n^{x-1}\left|a_{n}\right|^{x} \frac{\Gamma(n+1) \Gamma(1-y)}{\Gamma(n+2-y)}
\end{aligned}
$$

whence the conclusion of the theorem is immediate, since

$$
\frac{\Gamma(n+1)}{\Gamma(n+2-y)} \sim n^{y-1} \quad(n \rightarrow \infty) .
$$

Proof OF THEOREM 1 . It is enough to show that if

$$
\sum n^{\lambda-2}\left|a_{n}\right|^{\lambda}
$$

is finite, then $f \in H^{\lambda}$. For this purpose, set $x=\lambda$ and $y=0$ in Theorem 7. Noting that $M(r, f)<P(r, f), 0<r<1$, we see at once that $M(f)$ belongs 
to $L^{\lambda}(0,1)$. Hence, since $f$ is univalent, $f \in H^{\lambda}[8]$.

REMARK 1 . Since every univalent function on $\Delta$ satisfies (1.1) and (1.3) if $0<\lambda<\frac{1}{2}$, the argument just presented was really only necessary to deal with the range $\frac{1}{2} \leqslant \lambda<1$, and it may well be that the conclusion holds also, in this case, for all univalent functions.

5. Proofs of Theorems 2-4. Concerning Theorem 2, it is, of course, well known (see, for example, [4, p 87]) that $(a) \Rightarrow(b)$ for all $\lambda>0$ and that (a) $\Rightarrow$ (c) for all $\lambda \in(0,2]$. The inequality derived in the next theorem allows us to reverse these implications if $0<\lambda<1$ and $f$ belongs to $\mathcal{K}$.

THEOREM 8. Let $0<x<1$ and let $f \in \mathcal{K}$. Then, for $0<r<1$,

$$
r M_{x}^{x}\left(r, f^{\prime}\right)<\sec (\pi x / 2) \int_{0}^{r} M^{x}\left(t, f^{\prime}\right) d t
$$

Proof. Select a pair of functions $g \in \mathcal{S}^{*}$ and $h \in \mathcal{P}$ to satisfy (2.3) and then define $f_{x}$ by

$$
z f_{x}^{\prime}(z)=g_{x}(z) h_{x}(z), \quad z \in \Delta,
$$

where

$$
g_{x}(z)=z(g(z) / z)^{x} \in \mathcal{S}^{*}
$$

and

$$
h_{x}(z)=(h(z))^{x} \in \mathcal{P},
$$

so that $f_{x} \in \mathscr{K}$. Lemma 2 applies to $f_{x}$ and so, if $0 \leqslant r<1$,

$$
\int\left|g_{x}\left(r e^{i \theta}\right)\right| \operatorname{Re} h_{x}\left(r e^{i \theta}\right) d \theta \leqslant 2 \pi M\left(r, f_{x}\right) .
$$

But

and hence

$$
\operatorname{Re} h_{x}(z) \geqslant \cos (\pi x / 2)|h(z)|^{x}, \quad z \in \Delta,
$$

$$
\begin{aligned}
r M_{x}^{x}\left(r, f^{\prime}\right) & =\frac{r}{2 \pi} \int\left|f^{\prime}\left(r e^{i \theta}\right)\right|^{x} d \theta \\
& =\frac{r^{1-x}}{2 \pi} \int\left|g\left(r e^{i \theta}\right)\right|^{x}\left|h\left(r e^{i \theta}\right)\right|^{x} d \theta \\
& \leqslant \sec (\pi x / 2) \frac{r^{1-x}}{2 \pi} \int\left|g\left(r e^{i \theta}\right)\right|^{x} \operatorname{Re} h_{x}\left(r e^{i \theta}\right) d \theta \\
& =\frac{\sec (\pi x / 2)}{2 \pi} \int\left|g_{x}\left(r e^{i \theta}\right)\right| \operatorname{Re} h_{x}\left(r e^{i \theta}\right) d \theta \\
& \leqslant \sec (\pi x / 2) M\left(r, f_{x}\right) \\
& \leqslant \sec (\pi x / 2) \int_{0}^{r} M\left(t, f_{x}^{\prime}\right) d t \\
& =\sec (\pi x / 2) \int_{0}^{r} M^{x}\left(t, f^{\prime}\right) d t
\end{aligned}
$$

as required. 
CoRollary 8.1. If $f \in \mathcal{K}, 0<\lambda<1$, and $M\left(f^{\prime}\right) \in L^{\lambda}(0,1)$, then $f^{\prime} \in H^{\lambda}$. In other words, (b) $\Rightarrow(\mathrm{a})$.

The proof is obvious.

COROLlaRY 8.2. If $f(z)=\sum a_{n} z^{n} \in \mathcal{K}, 0<\lambda<1$ and $\sum n^{2 \lambda-2}\left|a_{n}\right|^{\lambda}$ is convergent, then $f^{\prime} \in H^{\lambda}$, i.e., (c) $\Rightarrow$ (a).

Proof. Since for any univalent function $f$ we have [7]

$$
r M\left(r, f^{\prime}\right) \leqslant 2 M(r, f)(1-r)^{-1}, \quad 0<r<1,
$$

an application of Theorem 7 with $x=y=\lambda$ shows that $M\left(f^{\prime}\right)$ belongs to $L^{\lambda}(0,1)$, whence the result follows.

Before embarking on the proofs of Theorems 3 and 4, which are of a negative character, it may be in order to recall a few general facts about Riesz products of the form

$$
\prod_{k=1}^{\infty}(1+\cos (n(k) \theta))
$$

where the positive integers $n(k)$ satisfy the condition

$$
n(k+1)>2 \mu(k), \quad \mu(k)=\sum_{j=1}^{k} n(j) .
$$

(For information about Riesz products not mentioned here, the reader is referred to [14].)

The $k$ th partial product of (5.2) can be expressed as a linear combination of cosines:

$$
p_{k}(\theta)=\prod_{j=1}^{k}(1+\cos (n(j) \theta))=1+\sum_{n=1}^{\mu(k)} c_{m} \cos m \theta ;
$$

and $p_{k+1}(\theta)$ is obtained by adding to $p_{k}(\theta)$ the polynomial $p_{k}(\theta) \cos (n(k+$ 1) $\theta$ ) all of whose terms are of rank $\geqslant n(k+1)-\mu(k)>\mu(k)$.

The numbers $\left(c_{m}\right)$ generated in this way are such that $0<c_{m}<1$ and

$$
c_{n(k)}=1, \quad k=1,2, \ldots
$$

Consider now the function $h$ defined on $\Delta$ by the power series

$$
h(z)=1+\sum c_{n} z^{n} .
$$

Since each $p_{k}(\theta)$ is a nonnegative partial sum of the real part of the trigonometric series $1+\sum c_{m} e^{i m \theta}$, it follows that $h \in \mathscr{P}$. Moreover, $c_{m} \nrightarrow 0$, by (5.3). After these few remarks, we are ready to prove

THEOREM 9. Let $\varphi$ be any positive function defined and increasing on $[0,1)$ and such that $\varphi(r) \rightarrow \infty$ as $r \rightarrow 1^{-}$. Then there exists a function $h(z)=1+$ 
$\Sigma c_{n} z^{n} \in \mathscr{P}$ with $c_{n} \geqslant 0$, such that

$$
M(r, h)=O(\varphi(r)) \quad\left(r \rightarrow 1^{-}\right),
$$

but for which $c_{n} \nrightarrow 0$.

Proof. Let the sequence $\left(r_{n}\right)$ be defined by

$$
\frac{\log \left(1-r_{n}\right)}{\log r_{n}}=n, \quad n>1,
$$

so that $\frac{1}{2}<r_{1}<r_{2}<\ldots<1$ and

$$
r_{n}^{n}=1-r_{n}, \quad n=1,2, \ldots
$$

Choose a sequence of positive integers $(n(k))$ such that $n(k+1) / n(k)>3$ and

$$
\varphi\left(r_{n}(k)\right)>2^{k+2}, \quad k>1 .
$$

Since

$$
2 \mu(k)=2 \sum_{j=1}^{k} n(j)<2 n(k) \sum_{j=0}^{\infty} 3^{-j}=3 n(k)
$$

the sequence $(n(k))$ generates, in the manner described above, a function $h(z)=1+\sum c_{n} z^{n} \in \mathcal{P}$ having the properties that $c_{n}>0$ and $c_{n} \nrightarrow 0$. It remains to show that $M(r, h)=O(\varphi(r))$. To this end, note that

$$
2^{k}=p_{k}(0)=1+\sum_{m=1}^{\mu(k)} c_{m}, \quad k>1 .
$$

Let $r_{n(1)}<r<1$ and choose $q$ such that

$$
r_{n(q)}<r<r_{n(q+1)}
$$

Then

$$
\begin{aligned}
h(r) & <h\left(r_{n(q+1)}\right)=1+\sum c_{m} r_{n(q+1)}^{m} \\
& <1+\sum_{m=1}^{n(q+1)} c_{m}+\sum_{m>n(q+1)} r_{n(q+1)}^{m} \\
& <1+\sum_{1}^{\mu(q+1)} c_{m}+r_{n(q+1)}^{n(q+1)} /\left(1-r_{n(q+1)}\right) \\
& =2^{q+1}+1<2^{q+2}<\varphi\left(r_{n(q)}\right)<\varphi(r),
\end{aligned}
$$

by (5.4), (5.6) and (5.5).

REMARK 2. Those familiar with Riesz products will recognize that the construction used in Theorem 9 provides an indirect method of manufacturing singular measures having good continuity properties as measured by their moduli of continuity. While the existence of such measures is well 
known (cf. [13] and the references therein), the method just described yields ones whose Fourier-Stieltjes coefficients are nonnegative and 'large' infinitely often.

Proof of Theorem 3. Without loss of generality, we can suppose given a positive $\varphi(r)$ increasing to infinity as $r \rightarrow 1^{-}$and such that

$$
\int \varphi(t) d t<\infty \text {. }
$$

Choose $h(z)=1+\Sigma c_{n} z^{n}$ in accordance with Theorem 9 and define $f$ by

$$
f(z)=z \exp \int_{0}^{z}(h(w)-1) / w d w=\sum a_{n} z^{n} .
$$

Then $f \in \mathcal{S}^{*}$ and, by (5.7), $f \in H^{\infty}$. Since the Taylor coefficients of $f$ and $h$ are related by the equations

$$
(n-1) a_{n}=c_{n-1}+\sum_{k=2}^{n-1} a_{k} c_{n-k}, \quad n>3,
$$

we see that the $a_{n}$ are nonnegative and such that $n a_{n+1}>c_{n}$. Thus $n a_{n} \nrightarrow 0$ and so $f^{\prime} \notin H^{1}$, while at the same time

$$
M\left(r, f^{\prime}\right)=O(\varphi(r)), \quad r \rightarrow 1,
$$

a consequence of the identity

$$
z f^{\prime}(z)=f(z) h(z), \quad z \in \Delta .
$$

Proof of Theorem 4. This time, let the function $h(z)=1+\sum c_{n} z^{n}$ correspond to the Riesz product $\Pi(1+\cos (n(k) \theta))$, generated by the sequence $n(k)=2^{2^{k}}$. As before, $c_{n} \nrightarrow 0$, but now the $c_{n}$ are small on the average, in the sense that

$$
\sum_{1}^{N} c_{m}=O(\log N)
$$

as can be seen very easily from (5.6).

Since $0<c_{n}<1$ and $\lambda \geqslant 1$ we deduce that

$$
T_{N}=\sum_{1}^{N} c_{m}^{\lambda}=O(\log N)
$$

A summation by parts now reveals that, as $m \rightarrow \infty$,

$$
\begin{aligned}
\sum_{1}^{m} k^{\lambda-2} c_{k}^{\lambda} & =\sum_{1}^{m} k^{\lambda-2}\left(T_{k}-T_{k-1}\right) \quad\left(T_{0}=0\right) \\
& =m^{\lambda-2} T_{m}+\sum_{1}^{m-1}\left[k^{\lambda-2}-(k+1)^{\lambda-2}\right] T_{k} \\
& =O\left(m^{\lambda-2} \log m\right)+O\left(\sum_{1}^{m} k^{\lambda-3} \log k\right)=O(1)
\end{aligned}
$$


since $1<\lambda<2$. Thus

$$
\sum n^{\lambda-2} c_{n}^{\lambda}<\infty .
$$

Next, define $f$ by (5.8), so that $f \in \mathcal{S}^{*}$ and

$$
f(z)=z \exp \sum c_{n} z^{n} / n \text {. }
$$

As we have just proved, the series $\Sigma c_{n} / n$, in particular, is convergent. Hence, so is $\sum a_{n}$.

Rewriting (5.9), we see that, if $\beta \geqslant 0$,

$$
\begin{aligned}
(n-1) a_{n} & =\sum_{1}^{n-1} a_{n-k} c_{k}, \quad n \geqslant 2, \\
& =\sum_{1}^{n-1} a_{n-k} k^{\beta} c_{k} k^{-\beta} \\
& <(n-1)^{\beta} \sum_{1}^{n-1} a_{n-k}\left(c_{k} k^{-\beta}\right),
\end{aligned}
$$

i.e.

$$
(n-1)^{1-\beta} a_{n}<\sum_{1}^{n-1} a_{n-k}\left(c_{k} k^{-\beta}\right), \quad n>2 .
$$

Applying first Hölder's inequality, with exponents $\lambda$ and $\lambda /(\lambda-1)$, to these inequalities, and then adding the new ones, we find that

$$
\sum(n-1)^{\lambda(1-\beta)} a_{n}^{\lambda}<\left(\sum a_{n}\right)^{\lambda} \sum c_{n}^{\lambda} n-\lambda \beta,
$$

a simple special case of the remarkable inequality proved by Hardy and Littlewood in [6]. Taking $\beta=(2-\lambda) / \lambda$, we now deduce that

$$
\sum n^{2 \lambda-2} a_{n}^{\lambda}<\infty
$$

although $f^{\prime} \notin H^{1}$, because $\left(n a_{n}\right)$ does not converge to zero.

6. Proofs of Theorems 5 and 6. In this section we examine to what extent the converse of Theorem $\mathrm{D}$ holds when $f \in \mathcal{K}$. The answer is furnished by Theorem 5, whose proof we proceed to give. So, let $f \in \mathcal{K} \cap H^{\lambda}, p=\lambda / \nu$ and $q=\lambda /(\lambda-\nu)$. Then $p$ and $q$ are conjugate exponents and $q \nu<1$, by assumption. Using inequality (5.1) together with Hölder's inequality we find that

$$
\begin{aligned}
\int\left\{\operatorname{tM}\left(t, f^{\prime}\right)\right\}^{\nu} d t & <2^{\nu} \int M^{\nu}(t, f)(1-t)^{-\nu} d t \\
& <2\left(\int M^{p \nu}(t, f) d t\right)^{1 / p}\left(\int(1-t)^{-q \nu} d t\right)^{1 / q} \\
& =2(1-q \nu)^{-1 / q}\left(\int M^{p \nu}(t, f) d t\right)^{1 / p}
\end{aligned}
$$


By Theorem A, the last displayed integral is finite. Hence $M\left(f^{\prime}\right)$ belongs to $L^{\nu}(0,1)$ and the first part of Theorem 5 is now seen to be another consequence of Theorem 8 .

To complete the proof we need an example of a function in $\mathcal{K} \cap H^{\lambda}$ for some $\lambda>0$ whose derivative is not an element of $H^{\mu}$, where $\mu=\lambda /(1+\lambda)$. To this end, consider the function

$$
\begin{aligned}
\varphi(z) & =(1-z) \log ^{2}(1-z) / z^{2} \\
& =(1-z)\left(\sum z^{n-1} / n\right)^{2}=1-\sum \varphi_{n} z^{n} .
\end{aligned}
$$

It is easily seen that $\varphi_{n} \geqslant 0$ and that $\Sigma \varphi_{n}=1$. Hence $\varphi \in \mathscr{P}$. Likewise, if $0<\varepsilon<1$, the functions $\varphi^{\varepsilon},(1-z)^{1-\varepsilon^{\varepsilon}}(z)$ and their reciprocals belong to $\mathcal{P}$ if the branches are appropriately chosen. With these qualifications, the function

$$
h(z)=\frac{1}{(1-z)}\left\{\frac{z}{-\log (1-z)}\right\}^{2 e}, \quad z \in \Delta,
$$

is a member of $\mathcal{P}$. Since also any regular branch of

$$
g(z)=\frac{z}{(1-z)^{\alpha}}, \quad z \in \Delta,
$$

is starlike, if $0 \leqslant \alpha \leqslant 2$, it follows that the function $f$ defined by

$$
f^{\prime}(z)=\frac{1}{(1-z)^{1+\alpha}}\left\{\frac{z}{-\log (1-z)}\right\}^{2 \varepsilon}
$$

belongs to $\mathcal{K}$, if $0 \leqslant \alpha \leqslant 2$ and $0 \leqslant \varepsilon \leqslant 1$. (This generalizes an example given in [5].)

Now choose $\alpha$ in $(0,1)$ and then $\varepsilon$ so that $\alpha<2 \varepsilon<1+\alpha$. The estimate

$$
M(r, f)=O\left((1-r)^{-\alpha} \log ^{-2 \varepsilon}\left(\frac{1}{1-r}\right)\right)
$$

shows that $M(f) \in L^{1 / \alpha}(0,1)$ and, therefore, $f \in H^{1 / \alpha}$. However, since

$$
M\left(r, f^{\prime}\right)>\frac{1}{(1-r)^{1+\alpha}}\left\{\frac{r}{-\log (1-r)}\right\}^{2 e}
$$

and $2 \varepsilon<1+\alpha$,

$$
M\left(f^{\prime}\right) \notin L^{1 /(1+\alpha)}(0,1),
$$

and, a fortiori $f^{\prime} \notin H^{1 /(1+\alpha)}$, by Theorem 2 . This concludes the proof.

In contrast to the result just established, it is interesting to note that, if we work with the class of starlike functions, then we do obtain a direct converse of Theorem D. This is the content of the necessity part of Theorem 6, which 
is one of the consequences of our last main result.

THEOREM 10. Let $f \in \mathcal{S}^{*}$ and let $\alpha=\alpha(f)$ denote the order of $f$ as defined by (2.2). Then (i) $f \in H^{\lambda}, 0<\lambda<\infty$, if and only if $\alpha \lambda<1$ and (ii) $f^{\prime} \in H^{\mu}$, $0<\mu<1$, if and only if $\mu(1+\alpha)<1$.

Proof. (i) Suppose $\alpha \lambda<1$. The defining relation (2.2) shows that $M(f)$ belongs to $L^{\lambda}(0,1)$, and so $f \in H^{\lambda}$, since $f$ is univalent.

Suppose, conversely, that $f \in H^{\lambda}$ and that $\alpha>0$. It can be shown [9] that there is a complex number $\omega$, with $|\omega|=1$, such that

$$
|f(z)| \geqslant 2^{\alpha-2}|z|\left|a_{1}\right| /|1-\bar{\omega} z|^{\alpha}, \quad z \in \Delta,
$$

whence it results that $(1-z)^{-\alpha} \in H^{\lambda}$. Hence $\alpha \lambda<1$.

(ii) Suppose $f^{\prime} \in H^{\mu}$. Then, by Theorem $\mathrm{D}, f \in H^{\mu /(1-\mu)}$ and so, by what we have just done, $\alpha \mu<1-\mu$, i.e., $\mu(1+\alpha)<1$.

Conversely, if $\mu(1+\alpha)<1$, then, by part (i) again, there exists $\varepsilon>0$ such that $f$ belongs to $H^{p}$, where $p=(1+\varepsilon) \mu /(1-\mu)$, and so, by Theorem 5 , $f^{\prime} \in H^{\mu}$.

The following is an immediate consequence of part (i), and we omit the proof which is obvious.

COROLlaRY 10.1. If $f$ is starlike, then $f$ is a member of every Hardy space if and only if $\alpha(f)=0$.

\section{REFERENCES}

1. J. G. Clunie, On schlicht functions, Ann. of Math. (2) 69 (1959), 511-519.

2. J. G. Clunie and F. R. Keogh, On starlike and convex schlicht functions, J. London Math. Soc. 35 (1960), 229-233.

3. J. G. Clunie and Ch. Pommerenke, On the coefficients of close-to-convex univalent functions, J. London Math. Soc. 41 (1966), 161-165.

4. P. L. Duren, Theory of $\boldsymbol{H}^{p}$ spaces Academic Press, New York, 1970.

5. P. J. Eenigenburg and F. R. Keogh, On the Hardy class of some univalent functions and their derivatives, Michigan Math. J. 17 (1970), 335-346.

6. G. H. Hardy and J. E. Littlewood, An inequality, Math. Z. 40 (1935), 1-40.

7. W. K. Hayman, Multivalent functions, Cambridge Univ. Press, Cambridge, 1958.

8. F. Holland and J. B. Twomey, On Hardy classes and the area function, J. London Math. Soc. 17 (1978), 275-283.

9. F. Holland and D. K. Thomas, On the order of a starlike function, Trans. Amer. Math. Soc. 158 (1971), 189-201.

10. W. Kaplan, On close-to-convex functions, Michigan Math. J. 1 (1952), 169-185.

11. A. J. Lohwater, G. Piranian and W. Rudin, The derivative of a schlicht function, Math. Scand. 3 (1955), 103-106.

12. Ch. Pommerenke, On starlike and convex functions, J. London Math. Soc. 37 (1962), 209-224.

13. Harold S. Shapiro, A class of singular functions, Canad. J. Math. 20 (1968), 1425-1431.

14. A. Zygmund, Trigonometric series. I, Cambridge Univ. Press, Cambridge, 1959.

Department of Mathematics, University College, Cork, Ireland 